\title{
Numerical and experimental study on energy-harvesting piezoelectric flags
}

\author{
Yifan Xia \\ LadHyX, École Polytechnique \\ UME, ENSTA-Paristech \\ Palaiseau, France \\ xia.yifan@ladhyx.polytechnique.fr
}

\author{
Sébastien Michelin \\ LadHyX, École Polytechnique \\ Palaiseau, France \\ sebastien.michelin@ladhyx.polytechnique.fr
}

\author{
Olivier Doaré \\ UME, ENSTA-Paristech \\ Palaiseau, France \\ olivier.doare@ensta-paristech.fr
}

\begin{abstract}
The spontaneous self-sustained flapping of a piezoelectric flag placed in a fluid flow may be used to produce electricity. This abstract presents a study of the coupled dynamics of such a fluid-solid-electric system, and analyzes the influence of resonant output circuits on the harvesting efficiency. Both numerical and experimental investigations show enhancement of harvesting efficiency induced by resonant circuit.
\end{abstract}

\section{INTRODUCTION}

Classical phenomena in fluid-solid interactions, such as vortex-induced vibrations [1] and flag flutter [2], are interesting candidates of flow energy harvesting mechanisms as these interactions may result in self-sustained periodic vibrations of the solid in an uniform flow, thus in permanent energy transfer between the fluid flow and the solid.

An innovative way to harvest energy using fluid-solid interactions is covering a flexible flag with piezoelectric materials, which, due to their ability to generate electrical charge when being deformed, have become increasingly attractive as a potential vibration energy harvester [3]. Placed in a flow, such a flag, called hereafter piezoelectric flag, would flap spontaneously and subsequently exhibits self-sustained periodic vibration once the surrounding flow exceeds a critical velocity. The feasibility and performance of harvesting the electrical charge by a resistive circuit was recently studied, and it was shown that both the stability of the system and the fluttering dynamics are influenced by electrical coupling [4], [5].

The periodicity of a flag's flapping motion inspires the use of resonant circuits which are able to match their natural frequency to that of the flag's motion, and therefore enhances the harvesting efficiency through resonance, which, via the inverse piezoelectric effect [3], is also likely to impact the flag's dynamics. In this work, we add an inductor to the previously mentioned resistive circuit, forming a LC loop, the simplest of resonant circuits. Its influences on both the energy harvesting and the flapping dynamics will be presented in this paper through numerical and experimental results

\section{MOdELING}

A cantilevered flag, whose surface is covered by pairs of piezoelectric patches connected with reversed polarity through the flag, is placed in an axial fluid flow with a constant velocity (Fig. 1a). The remaining electrodes of piezoelectric pairs are connected to the output circuits [4].
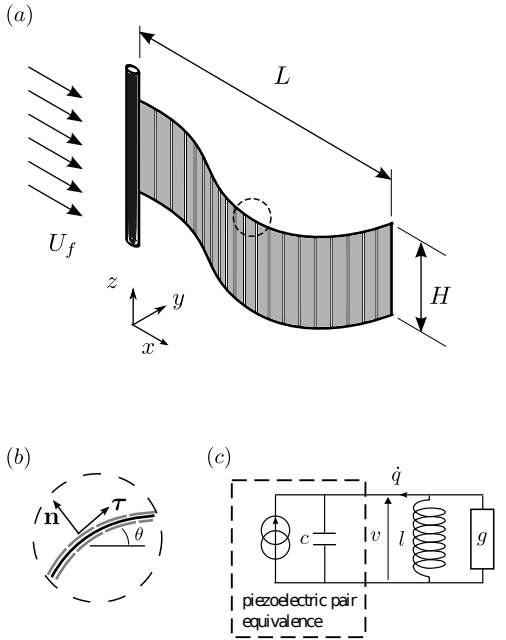

Fig. 1. Flapping piezoelectric flag in a uniform axial flow. (a): 3-dimension view; $(b)$ : zoom of the circled area in $(x, y)$ plane; $(c)$ : equivalent circuit of a piezoelectric pair connected with a parallel RL circuit.

Supposing continuous coverage of the flag by infinitesimal piezoelectric pairs, the electric state of the system is described using the local voltage $v$ and lineic charge transfer $q$, which are continuous functions of the streamwise Lagrangian coordinate $s$. The electrical circuits are characterized by a lineic conductance $g$, and a lineic inductive admittance 1/l (Fig. 1c).

We focus on the influence of the electrical circuit on the harvesting efficiency, which is formally defined as the ratio between the harvested energy, defined as the rate of dissipation in the resistive elements and the kinetic energy flux of the flow passing through the cross section occupied by the flag.[5]

\section{NUMERICAL RESUlTS}

The efficiency $\eta$ and flapping frequency $\omega$ are shown in Fig. 2. We observe that the efficiency is considerably increased for intermediate values of $\omega_{0}$ (Fig. 2a), a non-dimensional parameter characterizing the circut's natural frequency. For the same values of $\omega_{0}$, the flapping frequency locks to $\omega_{0}$ (Fig. $2 b$ ). We observe here a frequency lock-in phenomenon [6], which is classically reported in studies of Vortex-Induced Vibrations (VIV) [1]. Through this lock-in mechanism, the circuit, forced 


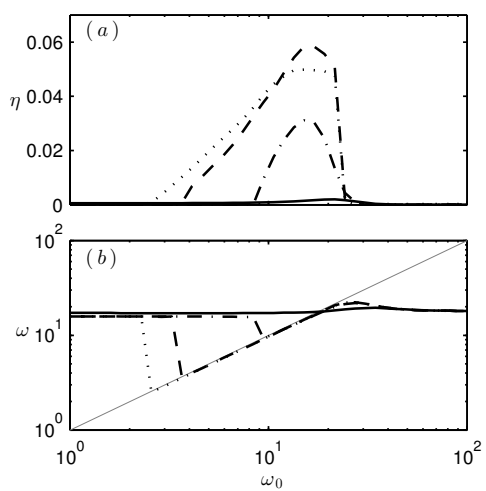

Fig. 2. (a) Harvesting efficiency $\eta$ and (b) flapping frequency $\omega$ as a function of $\omega_{0}$ for $\alpha=0.3, U^{*}=13$, and $\beta=0.05$ (solid), $\beta=1$ (dash-dot), $\beta=4$ (dashed), $\beta=8$ (dotted).

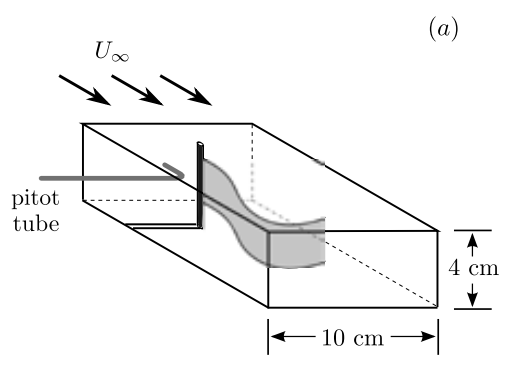

(b)

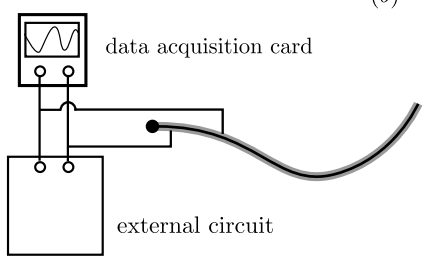

Fig. 3. Experimental setup: (a) a PVDF piezoelectric flag in the wind tunnel The flag is connected to an external circuit and measurement device $(b)$.

by the flag at its natural frequency, works permanently at resonance, yielding a high voltage, which leads to the increased harvesting efficiency.

\section{EXPERIMENTAL STUDY}

We conducted wind tunnel tests with a piezoelectric flag made of Polyvinylidene Difluoride (PVDF). Inside the test section, the flag is clamped at its upstream end by a mast fixed on the inner walls (Fig. 3a). The flag is connected to the output circuit, as well as a data acquisition board measuring the voltage (Fig. $3 b$ )

Preliminary measurements are performed and without the piezoelectric coupling, the flag's flapping frequency is obtained as $f=42.8 \mathrm{~Hz}$ at a flow velocity of $10.6 \mathrm{~m} / \mathrm{s}$. This frequency is chosen as the targeted frequency of the resonant circuit and, with the flag's capacitance, the inductance necessary to obtain a resonance is determined to be around $\mathcal{L} \sim 1000 \mathrm{H}$. This inductance is connected in parallel with a variable resistor, in

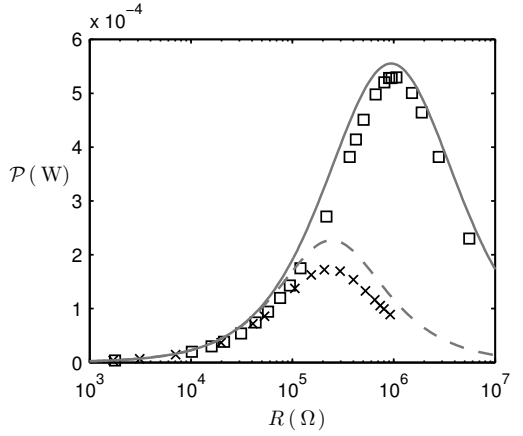

Fig. 4. Harvested power (markers) and model prediction (curves) using a PVDF flag without inductor (crosses, gray dashed) and with an inductor of $\mathcal{L}=1000 \mathrm{H}$ (squares, gray solid)

which the rate of dissipation $\mathcal{P}$ is considered as the harvested power.

We observe that when the inductive-resistive circuit is in resonance with the flapping flag (Figs. 4 squares, gray solid curve), the harvested power increases considerably compared with the purely resistive case (Figs. 4, crosses, gray dashed curve). These results suggest that the presence of inductance improves the energy harvesting performance by resonance. This resonance however has little effect on the flapping frequency (not shown), therefore the dynamics of the flags is marginally affected.

\section{CONCLUSION}

Our numerical results showed that through a frequency lock-in induced by the resonant circuit, the piezoelectric flag's harvesting performance is enhanced as well as the flapping dynamics is strongly impacted. Experimentally, the harvesting efficiency is increased due to resonance, yet little impact is observed in terms of the flapping dynamics, undoubtedly as a result of weak coupling obtained from PVDF.

\section{ACKNOWLEDGMENT}

This work was supported by the French National Research Agency ANR (Grant ANR-2012-JS09-0017). S. M. also acknowledges the support of a Marie Curie International Reintegration Grant within the 7th European Community Framework Programme (PIRG08-GA-2010-276762).

\section{REFERENCES}

[1] C. H. K. Williamson and R. Govardhan, "Vortex-induced vibrations," Annu. Rev. Fluid. Mech., vol. 36, no. 1, pp. 413-455, 2004

[2] M. J. Shelley and J. Zhang, "Flapping and bending bodies interacting with fluid flows," Annu. Rev. Fluid. Mech., vol. 43, pp. 449-465, 2011.

[3] A. Erturk and D. J. Inman, Piezoelectric energy harvesting. John Wiley \& Sons, 2011

[4] O. Doaré and S. Michelin, "Piezoelectric coupling in energy-harvesting fluttering flexible plates: linear stability analysis and conversion efficiency," J. Fluids Struct., vol. 27, no. 8, pp. 1357 - 1375, 2011.

[5] S. Michelin and O. Doaré, "Energy harvesting efficiency of piezoelectric flags in axial flows," J. Fluid Mech., vol. 714, pp. 489-504, 2013.

[6] Y. Xia, S. Michelin, and O. Doaré, "Fluid-solid-electric lock-in of energyharvesting piezoelectric flags," Phys. Rev. Applied, vol. 3, p. 014009, 2015 . 